\title{
Evidence for Bosonization in a Three-Dimensional Gas of SU $(N)$ Fermions
}

\author{
Bo Song $\odot,{ }^{1, * \S}$ Yangqian Yan $\odot,{ }^{2, *}$ Chengdong He, ${ }^{1}$ Zejian Ren, ${ }^{1}$ Qi Zhou, ${ }^{2,3, \uparrow}$ and Gyu-Boong Jo ${ }^{1, \$}$ \\ ${ }^{1}$ Department of Physics, The Hong Kong University of Science and Technology, \\ Clear Water Bay, Kowloon, Hong Kong, China \\ ${ }^{2}$ Department of Physics and Astronomy, Purdue University, West Lafayette, Indiana 47907, USA \\ ${ }^{3}$ Purdue Quantum Science and Engineering Institute, Purdue University, \\ 1205 West State Street, West Lafayette, Indiana 47907, USA
}

(Received 6 March 2020; accepted 9 November 2020; published 16 December 2020)

\begin{abstract}
Blurring the boundary between bosons and fermions lies at the heart of a wide range of intriguing quantum phenomena in multiple disciplines, ranging from condensed matter physics and atomic, molecular, and optical physics to high-energy physics. One such example is a multicomponent Fermi gas with $\mathrm{SU}(N)$ symmetry that is expected to behave like spinless bosons in the large- $N$ limit, where the large number of internal states weakens constraints from the Pauli exclusion principle. However, bosonization in $\operatorname{SU}(N)$ fermions has never been established in high dimensions where exact solutions are absent. Here, we report direct evidence for bosonization in a $\mathrm{SU}(N)$ fermionic ytterbium gas with tunable $N$ in three dimensions (3D). We measure contacts, the central quantity controlling dilute quantum gases, from the momentum distribution and find that the contact per spin approaches a constant with a $1 / N$ scaling in the low-fugacity regime consistent with our theoretical prediction. This scaling signifies the vanishing role of the fermionic statistics in thermodynamics and allows us to verify bosonization through measuring a single physical quantity. Our work delivers a highly controllable quantum simulator to exchange the bosonic and fermionic statistics through tuning the internal degrees of freedom in any generic dimensions. It also suggests a new route toward exploring multicomponent quantum systems and their underlying symmetries with contacts.
\end{abstract}

DOI: 10.1103/PhysRevX.10.041053

\section{INTRODUCTION}

Bosons and fermions exhibit intrinsically different properties because of the distinct underlying statistics. Strikingly, the boundary between bosons and fermions could become blurred under a variety of scenarios in condensed matter and high-energy physics [1-4], ranging from the supersymmetry exchanging bosons and fermions [3] to fermionization of strongly interacting bosons in 1D [1,2]. In the latter case, hard-core bosons and noninteracting fermions share identical thermodynamical properties despite the fact that their correlation functions are different $[5,6]$. Another interesting route is to increase the number of spin

\footnotetext{
*These authors contributed equally to this work.

† zhou753@purdue.edu

"gbjo@ust.hk

${ }^{\S}$ Present address: Cavendish Laboratory, University of Cambridge, Cambridge CB3 OHE, United Kingdom.

Published by the American Physical Society under the terms of the Creative Commons Attribution 4.0 International license. Further distribution of this work must maintain attribution to the author(s) and the published article's title, journal citation, and DOI.
}

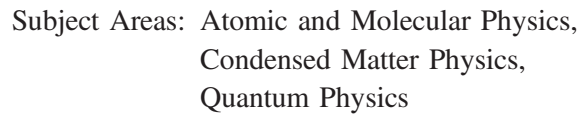

components $N$ in $\mathrm{SU}(N)$ fermions leading to bosonization [7]. Theoretically, such bosonization of $\mathrm{SU}(N)$ fermions has been extensively studied in 1D [7-14]. In this particular reduced dimension, exact solutions exist and allow one to confirm bosonization in the large- $N$ limit $[7,8]$. Experimentally, this phenomenon has also been explored in 1D, showing that the breathing mode of $\mathrm{SU}(N)$ fermions approaches that of bosons with increasing $N$ [15].

In spite of the aforementioned serious efforts of studying bosonization of $\mathrm{SU}(N)$ fermions, some fundamental questions about bosonization of $\mathrm{SU}(N)$ fermions remain unanswered so far. First, does bosonization of $\mathrm{SU}(N)$ fermions occur in high dimensions? Since exact solutions generically do not exist beyond $1 \mathrm{D}$, it is challenging to rigorously prove the bosonization in high dimensions. In addition, the breathing mode alone cannot tell whether other thermodynamic quantities approach those of bosons. In practice, it is difficult to measure all thermodynamic quantities. Therefore, is it possible to use a single quantity to establish bosonization?

In this work, we explore bosonization of a 3D $\mathrm{SU}(N)$ Fermi gas by measuring its central quantity, the so-called contact $\mathcal{C}$ [16-18], and answer both questions. Through celebrated universal relations, contacts govern other physical observables, such as the momentum distribution, 
the energy, the pressure, and a variety of spectroscopies [19-24]. Therefore, the dependence of contacts on $N$ directly provides us with the evidence of bosonization without resorting to measuring other thermodynamic quantities. We choose ${ }^{173} \mathrm{Yb}$ atoms as our sample, in which the number of internal states accessible in experiments is highly tunable, ranging from one to six. Because of the strong decoupling between electronic and nuclear spins, interactions between nuclear spins are isotropic, providing the many-body system with a $\mathrm{SU}(N)$ symmetry and, consequently, a wide range of exotic phenomena [25-28].

Whereas the $\mathrm{SU}(N)$ symmetry has been explored in optical lattices [15,29-31], a spectroscopy [32-34], and collective excitations $[15,35]$, it is still challenging to measure the rather small contact due to the weak interactions between ${ }^{173} \mathrm{Yb}$ atoms. To overcome this obstacle, we develop a new protocol to extract the contact from the column-integrated momentum distribution without using the inverse-Abel transform, which allows a high signal-tonoise ratio (SNR). We measure the temperature dependence of the contact when the temperature $T / T_{F}$ decreases from 1.0 to 0.55 and compare experimental results with theoretical calculations based on the virial expansion. Whereas the second-order virial expansion shows a scaling of $\left(T / T_{F}\right)^{-3 / 2}$, high-order virial coefficients lead to corrections from other powers of $T / T_{F}$. When $N$ is fixed, no change in the measured contact is observed for different spin constituents, confirming the isotropic interaction. We emphasize that the underlying mechanism for $\mathrm{SU}(N)$ fermions, the large internal degree of freedom weakening the Pauli exclusion principle, is the same for any temperature and any interaction strength. In different parameter regimes, the quantitative difference is how fast physical observables approach those of bosons. We, thus, focus on the temperature regime readily achievable in current experiments to deliver evidence for bosonization in three dimensions for the first time in laboratories, though the multicomponent nature of $\mathrm{SU}(N)$ fermions may allow a more efficient cooling down to even lower temperatures [36]. Since only a finite $N$ is accessible in realistic experiments, it is critical to work out and experimentally verify how physical observables scale with $N$ so as to access an unambiguous proof of bosonization in the large$N$ limit. To this end, we further change the number of nuclear spin components $N$ and keep the number of atoms per component constant at the same temperature and trap geometry. We find a linear dependence of the contact with $N$. Consequently, the contact per spin approaches a constant with a scaling law of $1-1 / N$.

\section{BOSONIZATION AND SCALING OF CONTACTS IN SU $(N)$ FERMIONS}

The observed scalings of contacts can be qualitatively understood as follows. As depicted in Fig. 1, in a balanced $\mathrm{SU}(N)$ gas with $N_{0}$ atoms per spin state, a single atom with

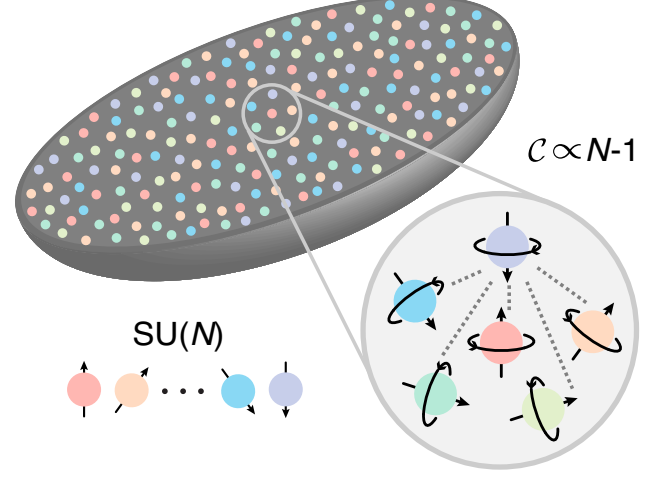

FIG. 1. Illustration of $s$-wave contacts in $\mathrm{SU}(N)$ fermions with tunable spin. Arrows with different colors and orientations denote the different nuclear spin states as large as $N=6$. Dashed lines represent pairs formed by two particles with different spins. Each pair contributes equally to the contact, which leads to $\mathcal{C} \propto N-1$.

spin $\sigma$ interacts with $(N-1) N_{0}$ atoms in the other $(N-1)$ spin components with spin $\sigma^{\prime}\left(\sigma^{\prime} \neq \sigma\right)$ through the $s$-wave scattering. When interactions are spin independent, each pair of atoms contributes an equal amount, $c_{\text {pair }}$, to the large momentum tail, $n_{3 \mathrm{D}}^{\sigma}(\vec{k})=\mathcal{C}_{0} / k_{3 \mathrm{D}}^{4}$, where $\vec{k}=\left(k_{x}, k_{y}, k_{z}\right)$ is a $3 \mathrm{D}$ momentum vector and its norm $k_{3 \mathrm{D}}=|\vec{k}|$ is much greater than $k_{F}$ and other microscopic momentum scales. In the low-fugacity regime, where three-body correlations are negligible, $\mathcal{C}_{0}=c_{\text {pair }}(N-1) N_{0}^{2}$, i.e., scaled with $(N-1)$ when the number of spins, $N$, is tuned. Correspondingly, if we consider the total momentum distribution $n_{3 \mathrm{D}}(\vec{k})=$ $\sum_{\sigma} n_{3 \mathrm{D}}^{\sigma}(\vec{k})$, we could define the total contact $\mathcal{C}_{\mathrm{SU}(N)}=$ $N \mathcal{C}_{0}=c_{\text {pair }} N(N-1) N_{0}^{2}$. Dividing $\mathcal{C}_{\mathrm{SU}(N)}$ by $N_{t}^{2}$, where $N_{t}=N N_{0}$ is the total particle number, we obtain that $\mathcal{C}_{\mathrm{SU}(N)} / N_{t}^{2}=c_{\text {pair }}(1-1 / N)$.

In our experiment, $p$-wave scatterings are negligible, as the current temperature regime is smaller than the barrier of the $p$-wave interaction [37]. We, therefore, treat SU(1) fermions as noninteracting systems. This is precisely the origin of the $1 / N$ factor in the scaling of $\mathcal{C}_{\mathrm{SU}(N)} / N_{t}^{2}$ with $N$. The Pauli exclusion principle suppresses the $s$-wave scattering between two atoms with the same spin, as well as their contributions to the $s$-wave contact. To make a comparison, we consider spinless bosons with the same $N_{t}, T$, and the same scattering length $a_{s}$. Though $c_{\text {pair }}$ is independent on statistics, all $N_{t}\left(N_{t}-1\right) / 2$ pairs of particles in spinless bosons contribute to contacts such that the high-momentum tail is written as $n_{B}(\vec{k})=\mathcal{C}_{B} / k_{3 \mathrm{D}}^{4}$, where $\mathcal{C}_{B}=c_{\text {pair }} N_{t}\left(N_{t}-1\right) \approx c_{\text {pair }} N_{t}^{2}$ for large $N_{0}$, as the momentum distribution of identical particles doubles that of distinguishable particles. We obtain $\mathcal{C}_{\mathrm{SU}(N)} / N_{t}^{2}=$ $\mathcal{C}_{B} / N_{t}^{2}(1-1 / N)$, which shows that the $s$-wave contact of $\mathrm{SU}(N)$ fermions approaches that of bosons with a $1 / N$ scaling. Since $\mathcal{C}_{0} / N=\left(\mathcal{C}_{\mathrm{SU}(N)} / N_{t}^{2}\right) N_{0}^{2}$, we use the contact per spin, $\mathcal{C}_{0} / N$, to capture this scaling with a fixed $N_{0}$. 


\section{RESULTS}

The experiment starts with degenerate fermions prepared in a crossed hybrid optical dipole trap (ODT) consisting of far-detuned 1064 and $532 \mathrm{~nm}$ laser light. A six-component Fermi gas of ${ }^{173} \mathrm{Yb}$ atoms, loaded from an intercombination magneto-optical trap, is evaporatively cooled down to the temperature of about $100 \mathrm{nK}$ in the ODT in $6 \mathrm{~s}$. Along with the evaporation, an arbitrary spin mixture with $N=1,2, \ldots, 6$ is prepared using optical pumping and blasting processes [38]. Next, we exponentially ramp up ODT to the final trap depth in $60 \mathrm{~ms}$ resulting in sufficiently large trap frequencies (see the Appendix for details). Finally, the momentum distribution after a $4 \mathrm{~ms}$ (TOF) expansion is recorded in the $k_{x}-k_{y}$ plane by absorption imaging along the $z$ direction using the resonant imaging light of ${ }^{1} S_{0} \rightarrow{ }^{1} P_{1}$ transition. We note that the measurement of contact at high momentum is negligibly affected by the finite expansion time [39]. In Fig. 2(b), a typical highmomentum tail is observed in the $\mathcal{S}$ profile after the systematic noise is filtered out [40].

Our schematic protocol, for the high-precision measurement of the contact, is based on the momentum distribution of the atomic cloud after the time-of-flight expansion as shown in Fig. 2. Typically, to measure contacts from the momentum distribution, the atomic profile recorded in the 2D plane, which represents a column-integrated momentum distribution, needs to be inverse-Abel transformed to a 3D momentum distribution. However, inverse-Abel transform often intensifies measurement noise and exacerbates the SNR, because it involves a derivative of the atomic distribution, which inevitably limits the capability to detect contacts in a weakly interacting $\mathrm{SU}(N)$ Fermi gas. To overcome this limitation, we develop a protocol to extract contacts directly based on the weight of the highmomentum tail from a 2D time-of-flight image without using the inverse-Abel transform.

When $k_{3 \mathrm{D}}$ is much greater than the inverse of the harmonic oscillator length and other microscopic momentum scales, $n_{3 \mathrm{D}}^{\sigma}(\vec{k})$ becomes isotropic in $3 \mathrm{D}$ and approaches $\mathcal{C}_{0} / k_{3 \mathrm{D}}^{4}$. Here, we use $\mathcal{C}_{0}$ to distinguish the original definition of contact from the scaled one, $\mathcal{C}$, used in our experiment. To be noted, in a spin-balanced Fermi gas with $N$ components, the atom density for each spin $n_{3 \mathrm{D}}^{\sigma}\left(k_{3 \mathrm{D}}\right)$ is identical. Hereafter, it is normalized such that $\int n_{3 \mathrm{D}}^{\sigma}\left(k_{3 \mathrm{D}}\right) d^{3} k_{3 \mathrm{D}}=1$ in our experiment. Correspondingly, the column-integrated momentum distribution $n^{\sigma}(k)=\int_{-\infty}^{\infty} n_{3 \mathrm{D}}^{\sigma}\left(k_{3 \mathrm{D}}\right) d k_{z}$, which follows $\int n^{\sigma}(k) 2 \pi k d k=1$. The momentum is normalized by the Fermi wave number $k_{F}=\sqrt{2 E_{F} m} / \hbar$ with the Fermi energy $E_{F}=\hbar \bar{\omega}\left(6 N_{0}\right)^{1 / 3}$. Here, $\bar{\omega}$ is the averaged trap frequency, $m$ is the mass of ${ }^{173} \mathrm{Yb}$, and $\hbar$ is the reduced Planck constant. Contact $\mathcal{C}$ can be experimentally determined from the high-momentum plateau of a term $\mathcal{S}=$ $2 / \pi \cdot k^{3} n^{\sigma}(k)$ as follows (see the Appendix):

$$
\mathcal{C}=\lim _{k \rightarrow \infty} \mathcal{S}(k)=\lim _{k \rightarrow \infty} \frac{2}{\pi} \cdot k^{3} n^{\sigma}(k)=\frac{\mathcal{C}_{0}}{(2 \pi)^{3} N_{0} k_{F}} .
$$

Here, $\mathcal{C}$ is naturally normalized by the atom number per spin $N_{0}$ and the Fermi wave number $k_{F}$. The key advantage of our protocol is that no transform gets involved, resulting in a high SNR ratio. To further diminish the noise of the atomic profile, we typically repeat the measurement approximately 100 times, obtain an averaged image as shown in Fig. 2(a), and then azimuthally average the momentum distribution
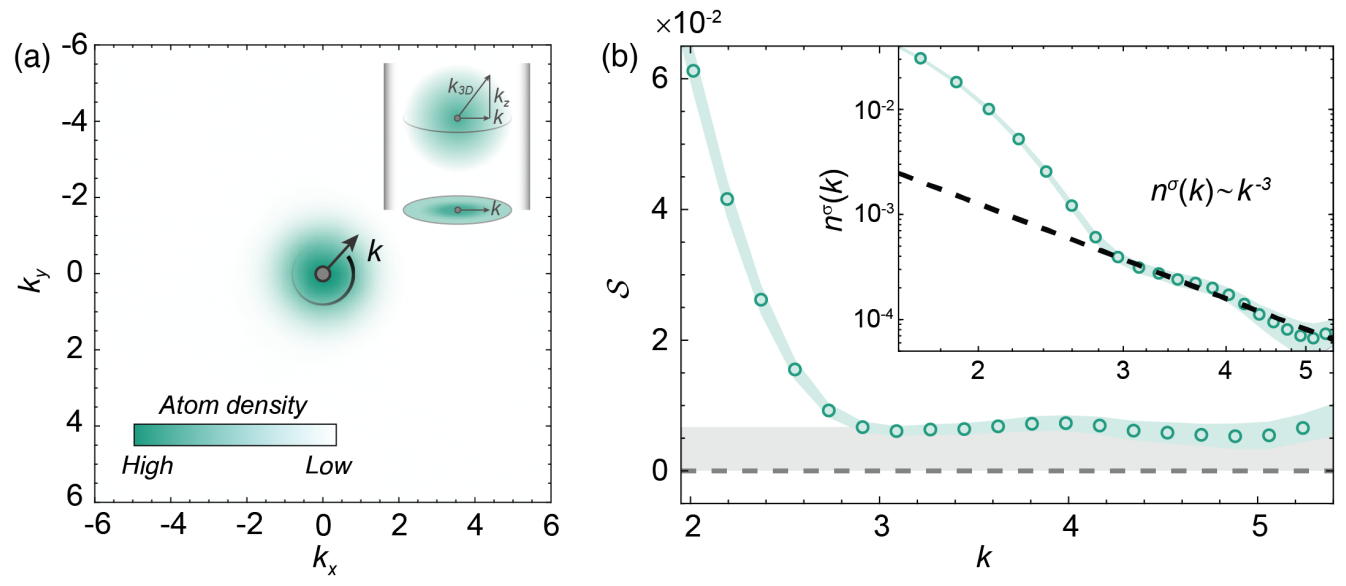

FIG. 2. Measurement of the contact parameter from the momentum distribution. (a) Momentum distribution of ${ }^{173}$ Yb atoms consisting of $N=6$ nuclear spin states after 4 ms time-of-flight expansion. Dimensionless momentum $k$ here is normalized by the Fermi wave number $k_{F}$. Note that momentum profile outside $k \simeq 2.5$ has already been subtracted by the momentum profile of the spin-polarized gas $(N=1)$ with the same total atom number. (b) $\mathcal{S}=2 / \pi \cdot k^{3} n^{\sigma}(k)$ is plotted as a function of momentum $k$ in units of $k_{F}$. The inset is the momentum tail of azimuthally averaged atomic distribution $n^{\sigma}(k)$ in logarithmic scale with a dashed guideline of $n^{\sigma}(k) \propto k^{-3}$. The $n^{\sigma}(k)$ is normalized as $\int n^{\sigma}(k) 2 \pi k d k=1$. The finite value of the contact is determined from the plateau of the $\mathcal{S}$ profile in the range of $k=3-4$. The green and gray shaded areas indicate the standard error and the measured value of contact, respectively. 
profile with $\pm 0.2 k_{F}$ moving average. To be noted, the value of the contact $\mathcal{C}$ measured by our protocol is in agreement with the result extracted from the 3D momentum distribution using the inverse Abel transform as described in the Appendix.

Because of the small scattering length of ${ }^{173} \mathrm{Yb}$, contacts in our $\mathrm{SU}(N)$ gas are contained in the large momentum tail with an extremely small amplitude that is below a thousandth of the maximum cloud density. To extract such a high-momentum tail from the subtle density profile, we first filter out the systematic noise (e.g., interference fringes, imaging light fluctuation) using the statistical method. Our protocol is based on statistical image decomposition and projection methods using the data images as a basis set and compensating for unwanted fringes [40]. Second, we compare the high-momentum tail of $\mathrm{SU}(N>1)$ fermions with respect to noninteracting $\mathrm{SU}(1)$ gases and extract the high-momentum tail of $\mathrm{SU}(N>1)$ gases after subtracting the counterpart of SU(1). This process allows us to systematically eliminate the diffraction effect arising from atoms. Note that, for a SU(1) gas, we first separate the dataset of SU(1) into two parts and analyze them using a similar procedure.

In Fig. 3, we show the measured $\mathcal{C}$ at temperatures between $T / T_{F}=0.55$ and $T / T_{F}=1$ for $\operatorname{SU}(N=1,3,6)$. We change the number of components, $N=1,2, \ldots, 6$ but keep the same number of atoms per spin component $N_{0}=6.7 \times 10^{3}$ in a 3D harmonic trap with frequencies $\left(\omega_{x}, \omega_{y}, \omega_{z}\right)=2 \pi \times(1400,750,250) \mathrm{Hz}$, the averaged trap frequency $\bar{\omega}=\left(\omega_{x} \omega_{y} \omega_{z}\right)^{1 / 3}=2 \pi \times 640 \mathrm{~Hz}$ and $k_{F} a_{s} \simeq 0.3$. We post-select data images according to the atom number and temperature with a tolerance of approximately $0.1 T_{F}$. As expected, a spin-polarized SU(1) gas with negligible $p$-wave scatterings does not exhibit a $k^{4}$ momentum tail within our experimental uncertainty, while the finite contact is clearly observed for a $\mathrm{SU}(6)$ or $\mathrm{SU}(3)$ Fermi gas in Fig. 3(a). Within the temperature regime we explore, the contact increases as the temperature $T / T_{F}$ decreases.

In Fig. 4, we test the scaling of the contact with the number of the spin components in $\mathrm{SU}(N)$ Fermi gases. We first collapse data points in Fig. 3(a) to the Fermi temperature using $\mathcal{C} \propto\left(T / T_{F}\right)^{-3 / 2}$ shown in Fig. 3(b). The results show that the temperature dependence of $\mathcal{C}$ is qualitatively consistent with the $\left(T / T_{F}\right)^{-3 / 2}$ scaling, a prediction from the second-order virial expansion (see the Appendix). However, high-order virial expansions lead to corrections to the temperature dependence of the contact, and both the third and the fourth virial coefficients $b_{3}$ and $b_{4}$, respectively, give rise to other powers of $T / T_{F}$ in the expression of the contact (see the Appendix). Such corrections are plotted in both Figs. 3(a) and 3(b). Since the result from the second-order virial expansion qualitatively captures temperature dependence and the current resolution in our experiment is not sufficient to accurately determine highorder corrections, we empirically extract the mean values of

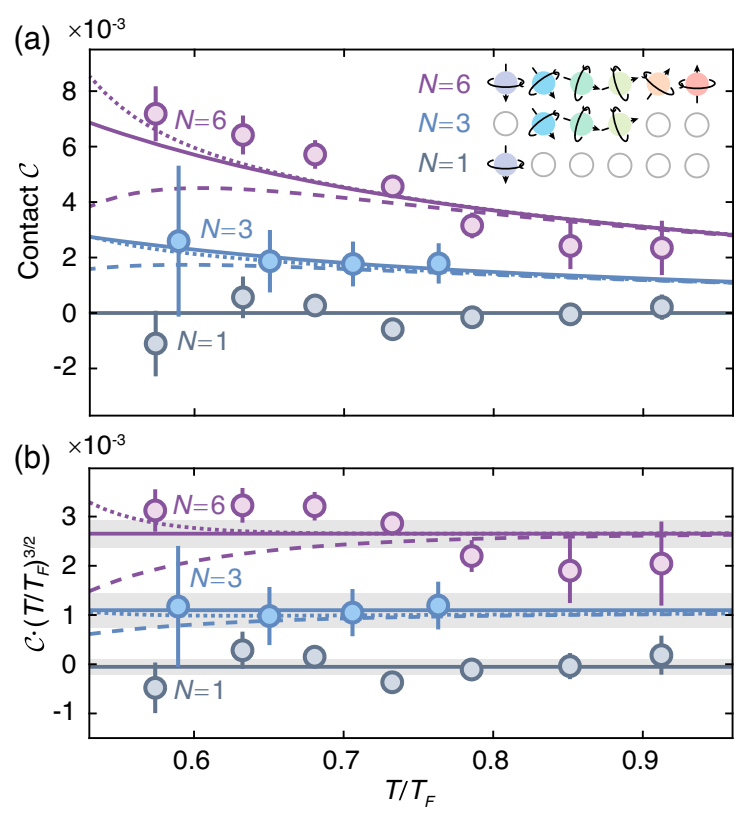

FIG. 3. Temperature scaling of contacts in $\mathrm{SU}(N)$ fermions. (a) Contacts are measured at different temperatures in the $\mathrm{SU}(N)$ Fermi gases. The error bars represent one standard deviation in the plateau area of $S$ profile. The solid curves are theoretical results multiplied by a factor of 7.5. Spin configuration of different $\mathrm{SU}(N)$ gases are presented by arrows in spheres, and the circle with nothing inside indicates the absence of the spin state. Details of spin configurations and preparation of different $\mathrm{SU}(N)$ gases are described in the Appendix. Solid curves represent the results from the second-order virial expansion $\mathcal{C} \propto\left(T / T_{F}\right)^{-3 / 2}$. Dashed and dotted curves represent results including corrections up to $b_{3}$ and $b_{4}$, respectively. (b) Using the temperature scaling $\mathcal{C} \propto\left(T / T_{F}\right)^{-3 / 2}$, contacts of $\mathrm{SU}(N)$ gases at different temperatures are collapsed to the Fermi temperature $T=T_{F}$. The solid lines are means of collapsed contacts. The shaded gray area indicates the experimental uncertainty, which consists of the standard error and the standard deviation of each point. Dashed and dotted theoretical curves including high-order corrections are no longer flat. A horizontal error bar of $\pm 0.05 T / T_{F}$ is not shown for every data point.

collapsed contacts to the Fermi temperature and further explore the dependence of the contact on $N$. Figure 4(a) shows that $\mathcal{C}$ depends linearly on $(N-1)$, and Fig. 4(b) demonstrates that $\mathcal{C} / N \sim \mathcal{C}_{0} / N$ approaches a constant with a $1 / N$ scaling. Because of the smallness of $a_{s} / \lambda$, where $\lambda=\sqrt{2 \pi \hbar^{2} /\left(m k_{B} T\right)}$ is the thermal wavelength, high-order virial expansions do not change the $1 / N$ scaling in the current parameter regime of our experiments (see the Appendix). All results are consistent with the qualitative picture we previously provided.

To measure $n^{\sigma}(k)$, we need to release atoms from the trap. Because of the absence of Feshbach resonance, interactions here cannot be turned off, unlike ${ }^{40} \mathrm{~K}$ for studying $s$-wave contacts of two-component fermions [21]. Interactions lead to complex expansion dynamics that are difficult to compute in theory. Therefore, it is illuminating to theoretically study 

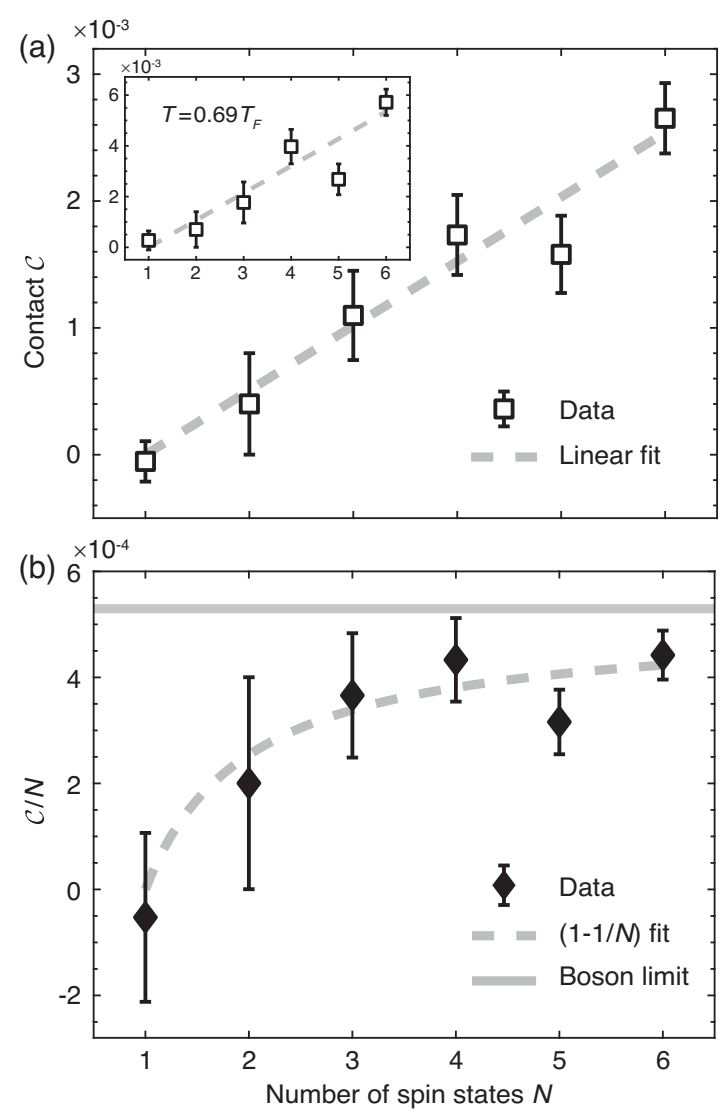

FIG. 4. Bosonization in $3 \mathrm{D} \operatorname{SU}(N)$ fermions. Scaling of the contact in $\mathrm{SU}(N)$ fermions with tunable spin is shown. (a) Contacts scale linearly with the number of spin components in a $\mathrm{SU}(N)$ Fermi gas. Mean values of collapsed contacts at $T=T_{F}$ of $\mathrm{SU}(N)$ gases are plotted as a function of the number of spin states. The dashed line is a linear fit to $(N-1)$. The inset shows contacts at $T=0.69 T_{F}$. (b) Contact per spin, $\mathcal{C} / N$, as a function of $N$. The dashed line indicates a $(1-1 / N)$ fit to the data. The horizontal solid line denotes the theoretical result of trapped bosons, $N_{0} \mathcal{C}_{B} /\left(8 \pi^{3} k_{F} N_{t}^{2}\right)$, multiplied by 7.5 .

contacts of trapped gases before the expansion. We compute contacts in the temperature regime explored in the experiments, $0.55 \leq T / T_{F} \leq 1.0$, where the second-order virial expansion works well and high-order virial expansions are negligible [41,42]. The virial expansion has been well established as a powerful tool to unfold fundamentally important principles using results at high temperatures, for instance, the universality of fermions at resonance $[43,44]$. Here, the spirit is the same. The virial expansion not only allows us to obtain quantitatively the $(1-1 / N)$ scaling in three dimensions where exact solutions generically do not exist, but also reveals the crucial role of the large internal degree of freedom in bosonization. We evaluate the local contact at the position $\vec{r}$ based on its local chemical potential $\mu_{\mathrm{loc}}=\mu_{0}-V(\vec{r})$, where $\mu_{0}$ is the chemical potential at the center of the trap and $V(\vec{r})$ is the harmonic trapping potential. The total contact is obtained by integrating local contacts in the trap. Using the second-order virial expansion, the contact is written as $\mathcal{C}_{0}=k_{B} T\left(8 \pi m / \hbar^{2}\right)\left(k_{B} T / \hbar \omega\right)^{3}$ $z^{2}\left(a_{s}^{2} / 2^{3 / 2} \lambda\right)(N-1) . \quad z=e^{\beta \mu_{\mathrm{loc}}}$ is the fugacity. In this temperature regime, the chemical potential $\mu_{0}$ is well approximated by $\mu_{0}=-T / T_{F} \cdot \log \left[6\left(T / T_{F}\right)^{3}\right] E_{F}$ [45]. We obtain

$$
\mathcal{C}=\frac{\mathcal{C}_{0}}{(2 \pi)^{3} N_{0} k_{F}} \approx \frac{m a_{s}^{2}}{6(2 \pi)^{5 / 2} \hbar^{2}} \frac{E_{F}}{\left(T / T_{F}\right)^{3 / 2}}(N-1) .
$$

We observe that $\mathcal{C}$ scales with $N-1$ and $\left(T / T_{F}\right)^{-3 / 2}$ in the high-temperature regime. In our experiments, $a_{s} / \lambda$ ranges between 0.06 and 0.08 . In such a weakly interacting regime, corrections from high-order virial coefficients modify the temperature dependence but not the scaling with $N$ (see the Appendix). Both scalings with $T$ and $N$ are consistent with the aforementioned experimental results, suggesting that interactions during the expansion do not change the scalings of the contact with $T$ and $N$.

Using the virial expansion, the $s$-wave contact of the spinless boson is also obtained explicitly in the same lowfugacity regime (see the Appendix). The second-order virial expansion leads to

$$
\mathcal{C}_{\mathrm{SU}(N)}=N \mathcal{C}_{0}=\left(1-\frac{1}{N}\right) \mathcal{C}_{B}
$$

As contact is the central quantity to control the many-body system, Eq. (3) is a direct proof of the bosonization without resorting to any other quantities, such as the full momentum distribution. Corrections from the third and the fourth virial coefficients introduce an extra temperature-dependent factor in Eq. (3) (see the Appendix).

Whereas scalings of the measured contacts with $N$ and $T$ after the expansion are consistent with theoretical results of trapped gases, experimental results lie systematically above theoretical ones, the former about 7.5 times greater than the latter (see the Appendix). It is interesting to note that such a discrepancy is also observed in an experiment measuring contacts of a weakly interacting Bose-Einstein condensate of ${ }^{4} \mathrm{He}$ atoms [46]. Interactions remain finite during expansions in both cases. It is, therefore, possible that interaction effects during the expansion lead to the aforementioned discrepancy. However, the current resolution limits our capability to measure the time dependence of contacts in the expansion, which by itself is an interesting question concerning the nonequilibrium dynamics of contacts. To avoid this issue and directly access contacts of trapped gases, an alternative scheme is the Bragg spectroscopy without the expansion [47].

\section{DISCUSSIONS AND CONCLUSION}

Whereas we focus on the high-temperature regime, it will be interesting to explore the low-temperature regime in the future. First, it is desirable to experimentally resolve 
corrections to the $1 / N$ scaling, which will allow us to understand how three-body and four-body correlations may affect contacts and bosonization. Second, with further decreasing $T$ down to below the superfluid transition temperature, our scheme of measuring contacts without using the inverse-Abel transform will provide us with an even richer playground to study contacts of superfluids with the $\mathrm{SU}(N)$ symmetry. It has been shown that contacts of superfluids are directly related to the superfluid order parameters $[48,49]$. It will be interesting to investigate how the interplay between the superfluid order and the $\mathrm{SU}(N)$ symmetry may bring us new macroscopic quantum phenomena and new universal relations governed by contacts, which may reveal deep connections between short-range correlations and many-body coherence.

It is worth mentioning that, due to the small scattering length of ytterbium, it is a difficult task to reach the exponentially small superfluid transition temperature. Though using other species with larger scattering lengths could increase the superfluid transition temperature, there are currently two $\mathrm{SU}(N)$ species with $N>2$ available in laboratories, ${ }^{173} \mathrm{Yb}$ and ${ }^{87} \mathrm{Sr}$. Unfortunately, the latter has an even smaller $a_{s}$ than the ${ }^{173} \mathrm{Yb}$ we are using now. As such, a more practical approach is to implement advanced cooling schemes, such as those engineering thermal reservoirs to absorb entropy from the system of interest [50,51]. Such schemes have been recently used to access long-range antiferromagnetic order in optical lattices [52]. It has also been recently used to access an extremely low entropy per particle of bosons down to $0.002 k_{b}$ in optical lattices [53]. Converting such entropy to the temperatures of ideal fermions leads to $T / E_{F} \sim 0.0004$, a temperature scale below the superfluid transition temperature of the weakly interacting ${ }^{173} \mathrm{Yb}\left(T / E_{F} \sim 0.005\right.$ for $\left.N=2\right)$. It is, thus, promising that superfluid transitions of $\mathrm{SU}(N)$ fermions will be accessible in the near future once the aforementioned cooling schemes or similar ones are implemented. In particular, the large spin degree of freedom may help $\mathrm{SU}(N)$ fermions to achieve even lower temperatures than spinless bosons, due to the Pomeranchuk effect [29]. It is then expected that utilizing similar schemes in $\mathrm{SU}(N)$ fermions will lead physicists to a new playground for exploring bosonization and many other exciting phenomena in the presence of both a large internal degree of freedom and symmetry breaking.

In addition to $\mathrm{SU}(N)$ fermions, our high-sensitivity measurement of contact in a microscopic level is also useful for other systems. For instance, contacts has been theoretically established as a powerful tool to explore deep connections between short-range correlations and manybody physics in spin-orbit coupled systems [54-56]. Since spin-orbit coupling is the fundamental mechanism behind topological quantum matters, we hope that our work will stimulate systematic studies of contacts in topological physics.

\section{ACKNOWLEDGMENTS}

G.-B. J. acknowledges the generous support from the Hong Kong Research Grants Council and the Croucher Foundation through GRF16311516 and GRF16305317, GRF16304918, GRF16306119, C6005-17G, N-HKUST601-17, and the Croucher Innovation grants, respectively. Q. Z. is supported by National Science Foundation PHY 1806796.

\section{APPENDIX: EXPERIMENTAL DETAILS AND THEORETICAL MODELS}

\section{Preparation of $\mathrm{SU}(N)$ gases}

$\mathrm{SU}(N)$ symmetric interaction in the ground state ${ }^{1} \mathrm{~S}_{0}$ of ${ }^{173} \mathrm{Yb}$ atoms emerges from the decoupling between nuclear spin and orbital angular momentum $(J=0)$. Exploiting the energy splitting of the excited state in ${ }^{3} P_{1}$ to our advantage, the narrow linewidth transition ${ }^{1} S_{0}(F=5 / 2) \rightarrow{ }^{3} P_{1}\left(F^{\prime}=7 / 2\right)$, with wavelength $\lambda=$ $556 \mathrm{~nm}$ and natural linewidth $\Gamma=2 \pi \times 181 \mathrm{kHz}$, is used as a blasting light to remove unwanted $m_{F}$ states of the ground manifold ${ }^{1} S_{0}$.

The preparation starts with a gas of spin-balanced six $m_{F}$ states which is initially loaded in an optical dipole trap. A sequence of short pulses of $\sigma^{ \pm}$optical blasting light resonance to transition $m_{F} \rightarrow m_{F} \pm 1$ is applied after the end of the evaporative cooling, where the temperature of atoms is $T \sim 100 \mathrm{nK}$. The magnetic field of $3.6 \mathrm{G}$ is applied, leading to a Zeeman splitting of $8.4 \mathrm{MHz} \sim 46 \Gamma$ between two adjacent $m_{F}$ states in the ${ }^{3} P_{1}$ state. Taking the preparation of a spin-balanced $\mathrm{SU}(2)$ gas as an example, we shine pulses of resonant blasting light with transitions $m_{F}=1 / 2 \rightarrow m_{F}^{\prime}=3 / 2$ and $m_{F}=3 / 2 \rightarrow m_{F}^{\prime}=5 / 2$ with $\sigma^{+}$polarization to remove positive $m_{F}=1 / 2$ and $m_{F}=3 / 2$, respectively, and $m_{F}=-1 / 2 \rightarrow m_{F}^{\prime}=-3 / 2$ and $m_{F}=-3 / 2 \rightarrow m_{F}^{\prime}=-5 / 2$ with $\sigma^{-}$polarization to remove negative $m_{F}=-1 / 2$ and $m_{F}=-3 / 2$, respectively, and the duration of each pulse is $5 \mathrm{~ms}$. Following a similar method, arbitrary spin configuration of the $\mathrm{SU}(N)$ $(N=1,2, \ldots, 6)$ gas can be prepared by the combination of $\sigma^{+}$and $\sigma^{-}$lights. The spin configurations of different $\mathrm{SU}(N)$ gases used in the experiment are detected by optical Stern-Gerlach effect [38] as shown in Fig. 5.

Notably, we use optical pumping to prepare spinpolarized gases $(N=1)$ with different atom numbers. At the beginning of evaporation, we first optically pump most of atoms to the $m_{F}=5 / 2$ state using another optical pumping light $400 \mathrm{MHz}$ red detuned from the resonance with ${ }^{1} S_{0} \rightarrow{ }^{1} p_{1}$ transition. The pumping pulse time is $300 \mathrm{~ms}$. Note that we intentionally leave other spin states for the sake of the evaporative cooling. At the end of the evaporation, all the other remained spin states are removed by $556 \mathrm{~nm}$ resonance light pulses similar to the procedure of $\mathrm{SU}(N)$ gas preparation. 


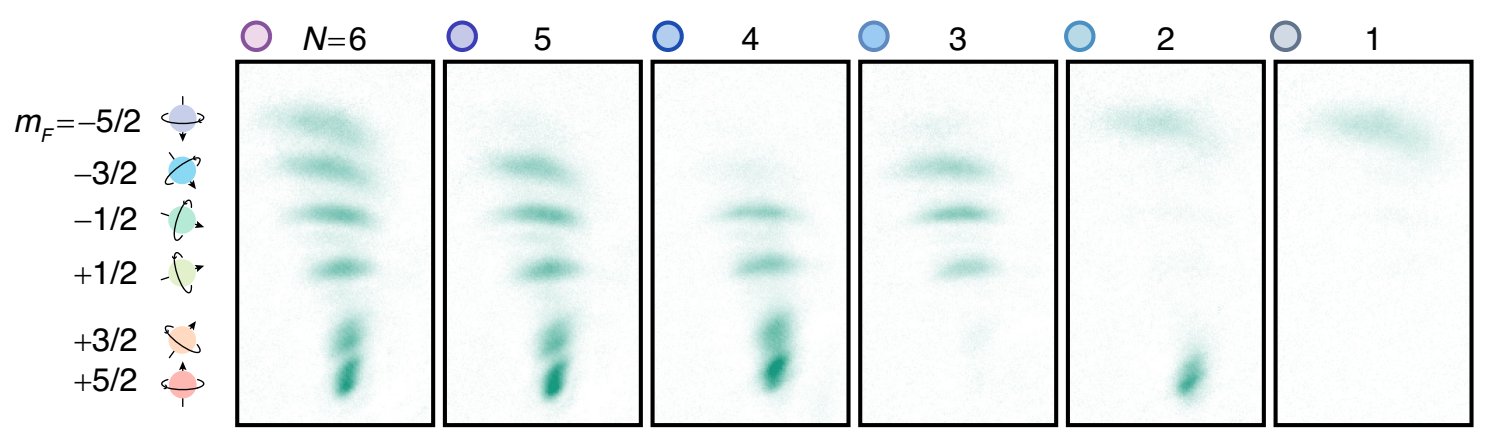

FIG. 5. Fermi gases with tunable spin components. Unwanted spin components are removed by short pulses of resonant $\sigma^{+}$and $\sigma^{-}$ atomic transitions from ${ }^{1} S_{0} \rightarrow{ }^{1} p_{1}$ in an around $13.6 \mathrm{G}$ magnetic field. From left to right, the number of spin states is prepared from $N=6$ to $N=1$. Optical Stern-Gerlach detection is used to monitor the spin configurations, and split subclouds from top to bottom are $m_{F}=-5 / 2$ to $m_{F}=5 / 2$.

We further increase the trap depth to obtain large trap frequency, after the preparation of degenerate Fermi gases with different spin components at the temperature of approximately $100 \mathrm{nK} . V(t)$, the trap depth of ODT, is increased exponentially from the initial $V_{i}$ to the final trap depth $V_{f}$ in $t_{f}=60 \mathrm{~ms}$ with a time constant $\tau=12 \mathrm{~ms}$ as follows:

$$
V(t)=a e^{t / \tau}+b,
$$

where $a=\left(V_{f}-V_{i}\right) /\left(e^{t_{f}} / \tau-1\right)$ and $b=V_{i}-a$. We experimentally test that $T / T_{F}$ values of both noninteracting gases $(N=1)$ and weakly interacting gases $(N=6)$ are conserved during the ODT is ramped up as shown in Fig. 6.

\section{Proof of the contact relation between $\mathcal{C}$ and $\mathcal{C}_{0}$}

Different from the original approach using the inverse Abel transform to get 3D normalized distribution $n_{3 \mathrm{D}}^{\sigma}(k)$ from 2D TOF image [21], the method demonstrated here is more robust against noise, because the contact is directly

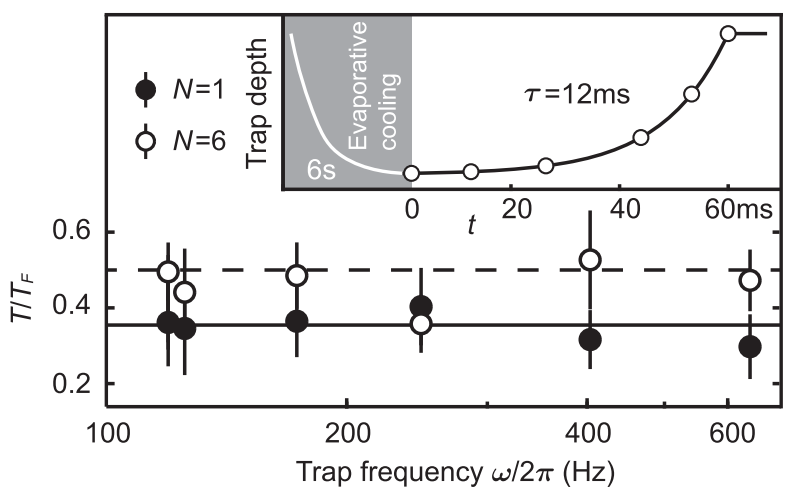

FIG. 6. The ramp-up of the optical dipole trap. $T / T_{F}$ of both noninteracting gases $(N=1)$ and weakly interacting gases $(N=6)$ are conserved during ramping up the optical dipole trap. The ODT is increased exponentially in $t_{\text {ramp }}=60 \mathrm{~ms}$ with the time constant $\tau=12 \mathrm{~ms}$ (inset). Gases with $N=1$ and $N=6$ components are initially prepared at $T=0.35 T_{F}$ and $0.5 T_{F}$, respectively. extracted based on the radial averaged atomic distribution $n^{\sigma}(k)$ from the 2D TOF image, illustrated in Fig. 2(a) in the main text. We calculate a term $\mathcal{S}=2 / \pi \cdot k^{3} n^{\sigma}(k)$ as a function of momentum $k$. The value of contact is experimentally extracted from the end tail of $\mathcal{S}$ profile. The contact $\mathcal{C}$ is, therefore, determined as $\mathcal{C}=\lim _{k \rightarrow \infty} \mathcal{S}$, which is slightly different from the original definition $\mathcal{C}_{0}[16,17]$. Contact defined here is naturally normalized by atom number per spin state $N_{0}$ and wave number $k_{F}$ and is associated with $\mathcal{C}_{0}$ as $\mathcal{C}=\mathcal{C}_{0} /\left[(2 \pi)^{3} N_{0} k_{F}\right]$. Here is the detail of the proof. In a spherical symmetry system which is confirmed experimentally, we start the derivation from the original definition of the contact $\mathcal{C}_{0}[16,17]$,

$$
\mathcal{C}_{0}=(2 \pi)^{3} N_{0} k_{F} \lim _{k_{3 \mathrm{D}} \rightarrow \infty} k_{3 \mathrm{D}}^{4} n_{3 \mathrm{D}}^{\sigma}\left(k_{3 \mathrm{D}}\right),
$$

where 3D wave vector $k_{3 \mathrm{D}}$ is normalized by $k_{F}$ and 3D density $n_{3 \mathrm{D}}^{\sigma}\left(k_{3 \mathrm{D}}\right)$ is normalized such that $\int n_{3 \mathrm{D}}^{\sigma}\left(k_{3 \mathrm{D}}\right) d^{3} k_{3 \mathrm{D}}=1$. The contact $\mathcal{C}$ defined in this article is written as

$$
\begin{aligned}
\mathcal{C} & =\lim _{k \rightarrow \infty} \mathcal{S} \\
& =\lim _{k \rightarrow \infty} 2 / \pi \cdot k^{3} n^{\sigma}(k) \\
& =2 / \pi \lim _{k \rightarrow \infty} k^{3} \int_{-\infty}^{\infty} n_{3 \mathrm{D}}^{\sigma}\left(k_{3 \mathrm{D}}\right) d k_{z} .
\end{aligned}
$$

Here, we substitute the radial averaged atomic density with $n^{\sigma}(k)=\int_{-\infty}^{\infty} n_{3 \mathrm{D}}^{\sigma}\left(k_{3 \mathrm{D}}\right) d k_{z}$. From Eq. (A2), for large $k_{3 \mathrm{D}}$, the 3D atomic density can be expressed as $n_{3 \mathrm{D}}^{\sigma}=\mathcal{C}_{0} /\left[(2 \pi)^{3} N_{0} k_{F}\right] \cdot k_{3 \mathrm{D}}^{-4}+\mathcal{O}\left(k_{3 \mathrm{D}}^{-5}\right)$, in which $\mathcal{O}\left(k_{3 \mathrm{D}}^{-5}\right)$ is the higher-order term. Substituting $n_{3 \mathrm{D}}^{\sigma}$ into Eq. (A3), with wave vector relation $k_{3 \mathrm{D}}^{2}=k^{2}+k_{z}^{2}$,

$$
\begin{aligned}
\mathcal{C} & =\frac{2 \mathcal{C}_{0}}{\pi(2 \pi)^{3} N_{0} k_{F}} \lim _{k \rightarrow \infty} k^{3} \int_{-\infty}^{\infty} \frac{d k_{z}}{\left(k^{2}+k_{z}^{2}\right)^{2}} \\
& =\frac{\mathcal{C}_{0}}{(2 \pi)^{3} N_{0} k_{F}} .
\end{aligned}
$$



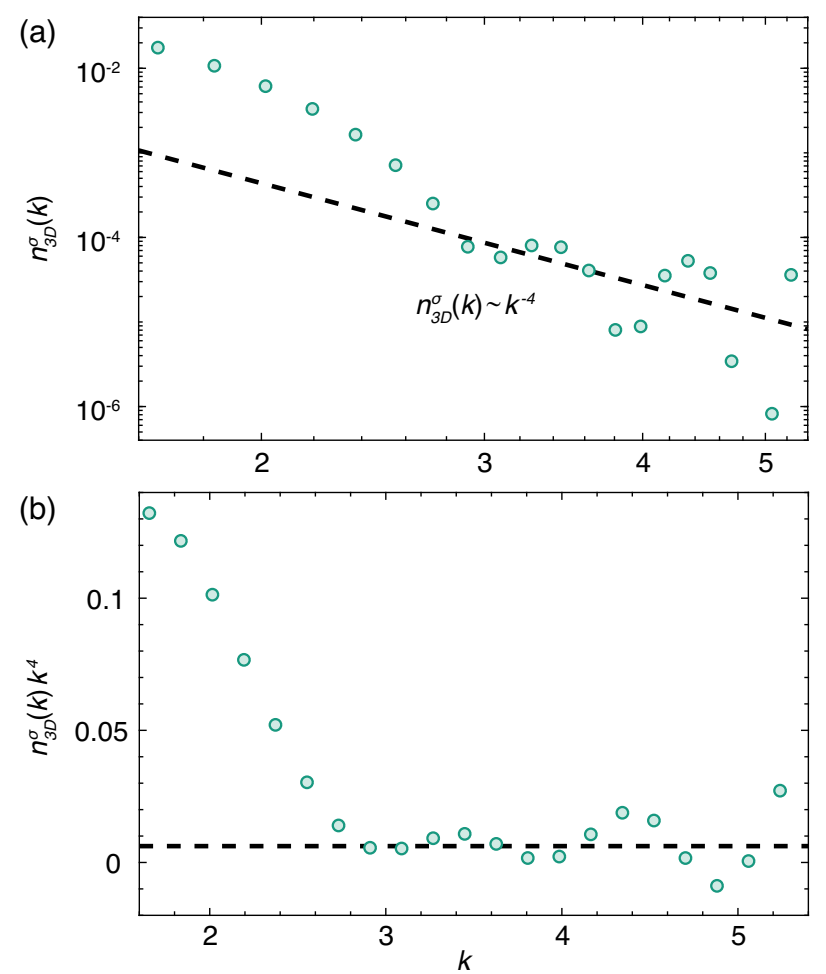

FIG. 7. Extracting the contact from the 3D momentum distribution. (a) 3D momentum distribution of a $\mathrm{SU}(6)$ gas is obtained from the column-integrated distribution (the same data in Fig. 1 in the main text) using an inverse Abel transform. The 3D density in high-momentum $k$ follows the power law $n_{3 \mathrm{D}}^{\sigma}(k) \sim k^{-4}$, which gives the contact $\mathcal{C}=0.007(1)$ with $95 \%$ confidence. (b) The contact is extracted from the tail of the term $n_{3 \mathrm{D}}^{\sigma}(k) k^{4}$ for $3<k<4$. The mean (one standard deviation in parentheses) is $0.006(3)$. The value of the contact extracted from the $3 \mathrm{D}$ momentum distribution is in agreement with the result of our method $0.0067(5)$ but has a larger uncertainty.

It is worth noting that we assume the momentum distribution is integrated over the momentum $k_{z}$ in Eqs. (A3) and (A4). However, the true momentum distribution along the $k_{z}$ can be slightly perturbed by atomatom interactions during the expansion. If this is the case, the measured contact $\mathcal{C}$ may be proportional to Eq. (6) with an unknown factor. To investigate this effect, we extract the value of contact from the three-dimensional density distribution using the inverse Abel transform in Fig. 7, which does not require any approximation used in Eqs. (A3) and (A4). The contact measured from the threedimensional density distribution is in good agreement with our result, validating our detection method.

\section{Theoretical model of contacts of $\mathrm{SU}(N)$ fermions}

In the grand-canonical ensemble, the thermodynamic potential $\Omega$ for $\mathrm{SU}(N)$ fermions can be expanded as a Taylor series at fugacity $z$ (virial expansion):

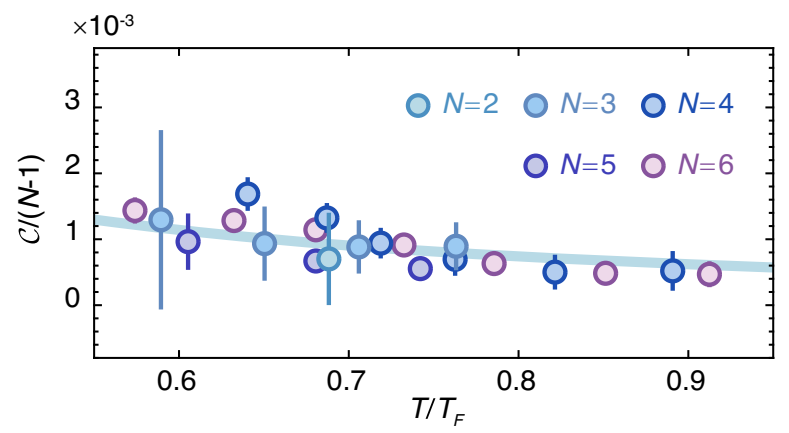

FIG. 8. Scaled contact vs temperature. Contact of different $\mathrm{SU}(N)$ gases is scaled on the $N=2$ components case by $\mathcal{C} /(N-1)$. The solid curve is the theoretical simulation multiplied by a factor of 7.5.

$$
\begin{aligned}
\Omega= & -k_{B} T Q_{1}(T)\left[N z+N b_{2}^{\mathrm{ni}} z^{2}\right. \\
& \left.+\frac{N(N-1)}{2} b_{2}\left(T, a_{s}\right) z^{2}+\cdots\right],
\end{aligned}
$$

where $Q_{1}(T)$ is the single-particle partition function, $b_{2}^{\text {ni }}$ is the intraspecies second-order virial coefficient which purely arises from particle statistics, and $b_{2}$ is the interspecies second-order virial coefficient which typically depends on the scattering length and temperature. Using the adiabatic relation [17]

$$
\left[\frac{\partial \Omega}{-a_{s}^{-1}}\right]_{T, \mu}=\frac{\hbar^{2} N}{8 \pi m} \mathcal{C}_{0}
$$

we obtain an virial expansion of the contact from Ref. [57]:

$$
\mathcal{C}_{0}=k_{B} T \frac{4 \pi m}{\hbar^{2}} Q_{1}(T) z^{2} \frac{\partial b_{2}\left(T, a_{s}\right)}{\partial a_{s}^{-1}}(N-1) .
$$

Comparing the Taylor expansion of the grand canonical potential $\Omega$ with the virial coefficient, we obtain

$$
b_{2}\left(a_{s}, T\right)=Q_{1,1} / Q_{1},
$$

where $Q_{1,1}$ is the partition function of two particles of different species in the anisotropic trap. According to Ref. [58], one could transform the problem, to a very good approximation at high temperature, to a spherical harmonic trap where trapping frequency $\tilde{\omega}$ satisfies

$$
3 \tilde{\omega}^{2}=\omega_{x}^{2}+\omega_{y}^{2}+\omega_{z}^{2} .
$$

Using the energy spectrum of two particles under isotropic harmonic confinement at any scattering length obtained from solutions in Ref. [59], we can numerically determine the partition function as well as the derivative with respect to $a_{s}^{-1}$. 
According to the local density approximation, at high temperature where $k_{B} T \gg \hbar \bar{\omega}$, the virial coefficient for the trapped system can be related to that of the homogeneous system:

$$
\begin{aligned}
& b_{2} \approx b_{2}^{\text {homo }} / 2^{3 / 2}, \\
& Q_{1} \approx\left(k_{B} T / \hbar \bar{\omega}\right)^{3},
\end{aligned}
$$

where $\bar{\omega}^{3}=\omega_{x} \omega_{y} \omega_{z}$. From Ref. [43], one obtains $b_{2}^{\text {homo }}=$ $-2 a_{s} / \lambda$, where $\lambda$ is the thermal de Broglie length. Combining the equations above, under local density approximation, we obtain

$$
\mathcal{C}_{0}=k_{B} T \frac{4 \pi m}{\hbar^{2}}\left(k_{B} T / \hbar \bar{\omega}\right)^{3} z^{2} \frac{2 a_{s}^{2}}{2^{3 / 2} \lambda}(N-1) .
$$

In the high-temperature limit, $z=N_{0}\left(\hbar \bar{\omega} / k_{B} T\right)^{3}$. The total contact of $\mathrm{SU}(N)$ fermions is then written as

$\mathcal{C}_{\mathrm{SU}(N)}=N \mathcal{C}_{0}=N_{0}^{2} N(N-1) \frac{2 \sqrt{2} \pi m}{\hbar^{2}} \frac{(\hbar \bar{\omega})^{3}}{\left(k_{B} T\right)^{2}} \frac{a_{s}^{2}}{\lambda}$.

The $(N-1)$ dependence of measured contact is shown in Fig. 8.

\section{Contact of single-component Bose gas}

Applying the virial expansion to a single-component Bose gas, the thermodynamic potential $\Omega_{B}$ at high temperatures is written as

$$
\Omega_{B}=-k_{B} T Q_{1}(T)\left[z_{B}+b_{2}^{\mathrm{niB}} z_{B}^{2}+b_{2}\left(T, a_{s}\right) z_{B}^{2}+\ldots\right] .
$$

Here $b_{2}$ is the second order virial coefficient for two distinguishable particles, i.e., the same as that for the intraspecies $b_{2}$ for $\mathrm{SU}(N)$ fermions, and $b_{2}^{\text {niB }}$ is a term that accounts for bosonic statistics which is independent of the scattering length. Using the adiabatic relation [17],

$$
\left[\frac{\partial \Omega_{B}}{-a_{s}^{-1}}\right]_{T, \mu}=\frac{\hbar^{2}}{8 \pi m} \mathcal{C}_{B}
$$

we obtain a virial expansion of the contact:

$$
\mathcal{C}_{B}=k_{B} T \frac{8 \pi m}{\hbar^{2}} Q_{1}(T) z^{2} \frac{\partial b_{2}\left(T, a_{s}\right)}{\partial a_{s}^{-1}} .
$$

Using $z_{B}=N N_{0}\left(\hbar \bar{\omega} / k_{B} T\right)^{3}$, we obtain

$$
\mathcal{C}_{B}=\left(N_{0} N\right)^{2} \frac{2 \sqrt{2} \pi m}{\hbar^{2}} \frac{(\hbar \bar{\omega})^{3}}{\left(k_{B} T\right)^{2}} \frac{a_{s}^{2}}{\lambda} .
$$

Comparing $\mathcal{C}_{B}$ and $\mathcal{C}_{\mathrm{SU}(N)}$, we obtain

$$
\mathcal{C}_{\mathrm{SU}(N)}=\frac{N-1}{N} \mathcal{C}_{B}
$$

In the limit $N \rightarrow \infty, \mathcal{C}_{\mathrm{SU}(N)}$ approaches $\mathcal{C}_{B}$ with a scaling of $1 / N$.

\section{High-order virial expansions}

With decreasing temperature, high-order virial expansions are required. We consider corrections up to $b_{4}$, i.e.,

$$
\begin{aligned}
\Omega= & -k_{B} T Q_{1}(T)\left[N z+N b_{2}^{\text {ni }} z^{2}+\frac{N(N-1)}{2} b_{2}\left(T, a_{s}\right) z^{2}\right] \\
& -k_{B} T\left(\Omega_{3} z^{3}+\Omega_{4} z^{4} \ldots\right),
\end{aligned}
$$

where

$$
\begin{aligned}
\Omega_{3}= & \left(\begin{array}{c}
N \\
3
\end{array}\right) Q_{1,1,1}+2\left(\begin{array}{c}
N \\
2
\end{array}\right) Q_{1,2}+N Q_{3} \\
& -N Q_{1}\left[N Q_{2}+\left(\begin{array}{c}
N \\
2
\end{array}\right) Q_{1,1}\right]+N^{3} Q_{1}^{3} / 3,
\end{aligned}
$$

$$
\begin{aligned}
\Omega_{4}= & N(N-1) Q_{1,3}+\left(\begin{array}{c}
N \\
2
\end{array}\right) Q_{2,2}+\left(\begin{array}{c}
N-1 \\
2
\end{array}\right) N Q_{1,1,2} \\
& +\left(\begin{array}{c}
N \\
4
\end{array}\right) Q_{1,1,1,1}+N Q_{4} \\
& -N Q_{1}\left[\left(\begin{array}{c}
N \\
3
\end{array}\right) Q_{1,1,1}+N Q_{3}+N(N-1) Q_{1,2}\right] \\
& -\frac{1}{2}\left[\left(\begin{array}{c}
N \\
2
\end{array}\right) Q_{1,1}+N Q_{2}-N^{2} Q_{1}^{2}\right]^{2}+\frac{1}{2} N^{4} Q_{1}^{4} .
\end{aligned}
$$

Here, $\left(\begin{array}{l}N \\ k\end{array}\right)=N ! /[k !(N-k) !]$ is the binomial coefficient, $Q_{n_{1}, n_{2} \ldots, n_{N}}$ means the partition function of the $N$-component fermionic system with $n_{i}$ number of fermions in the $i$ th component, and the order of $n_{1}, n_{2} \ldots, n_{N}$ does not matter because all spins are equivalent. For instance, $Q_{1,2}$ means the partition function of three fermions with one particle in one spin state and two particles in another one. When each of the three fermions occupies a different spin state, its partition function is denoted by $Q_{1,1,1}$.

In our experiment, $a_{s} / \lambda$ is a small number between 0.06 and 0.08. In such a weakly interaction regime, where $\left(a_{s} / \lambda\right)^{2} \ll 1, Q_{1,1,1}=Q_{1}^{3}+3\left(Q_{1,1}-Q_{1}^{2}\right) Q_{1}+O\left[\left(a_{s} / \lambda\right)^{2}\right]$. This result can be understood from the fact that the partition function of three fermions, all of which occupy different spin states, reduces to $Q_{1}^{3}$ in the noninteracting limit. Turning on a weak interaction, the leading correction is $3\left(Q_{1,1}-Q_{1}^{2}\right) Q_{1} \sim a_{s} / \lambda$; i.e., these three fermions can be decomposed to a pair of particles, which are interacting with the two-body interaction, and a remaining one that 
does not interact with the pair. The factor of 3 comes from the three ways of such decomposition. We thus obtain

$$
\begin{aligned}
\Omega_{3}= & 2\left(\begin{array}{c}
N \\
2
\end{array}\right)\left(Q_{1,2}-Q_{1} Q_{2}-Q_{1,1} Q_{1}+Q_{1}^{3}\right) \\
& +N\left(Q_{3}-Q_{1} Q_{2}+Q_{1}^{3} / 3\right) .
\end{aligned}
$$

Similar to what we see in the second-order virial expansion, $\Omega_{3}$ can also be separated into two parts: One is the result of noninteracting systems, and other is the correction from interactions. We thus rewrite the above equation as

$$
\Omega_{3} / Q_{1}=N(N-1) b_{3}^{\mathrm{int}}+N b_{3}^{\mathrm{ni}},
$$

where $b_{3}^{\text {int }} \equiv\left(Q_{1,2}-Q_{1} Q_{2}-Q_{1,1} Q_{1}+Q_{1}^{3}\right) / Q_{1}$ and $b_{3}^{\text {ni }} \equiv$ $\left(Q_{3}-Q_{1} Q_{2}+Q_{1}^{3} / 3\right) / Q_{1}$. Again, the noninteracting part of $b_{3}$ is proportional to $N$, while the contribution from interactions is proportional to $N(N-1)$. Higher powers of $N$, such as $N(N-1)(N-2)$, which is at least of the order of $\left(a_{s} / \lambda\right)^{2}$, are high-order corrections and, thus, are negligible in the weakly interacting regime.

The same analyses can be applied to $b_{4}$. In the weakly interacting regime, $a_{s} / \lambda \ll 1$, the partition functions of more than three spin components can be expressed in terms of those of two- and one-spin components. We thus obtain

$$
\begin{aligned}
-\frac{\Omega}{N k_{B} T Q_{1}}= & z+\frac{1}{2} z^{2}\left[(N-1) b_{2}^{\mathrm{int}}+b_{2}^{\mathrm{ni}}\right] \\
& +\frac{1}{3} z^{3}\left[3(N-1) b_{3}^{\mathrm{int}}+b_{3}^{\mathrm{ni}}\right] \\
& +\frac{1}{4} z^{4}\left[4(N-1) b_{4}^{\mathrm{int}}+b_{4}^{\mathrm{ni}}\right]+\cdots,
\end{aligned}
$$

where $b_{n}$ are expanded in terms of $a_{s} / \lambda$ :

$$
\begin{gathered}
b_{2}^{\mathrm{ni}}=-1 / 8, \\
b_{2}^{\mathrm{int}}=-\frac{a_{s}}{\sqrt{2} \lambda}+O\left(\frac{a_{s}}{\lambda}\right)^{2}, \\
b_{3}^{\mathrm{ni}}=1 / 27, \\
b_{3}^{\mathrm{int}}=\frac{a_{s}}{3 \sqrt{6} \lambda}+O\left(\frac{a_{s}}{\lambda}\right)^{2}, \\
b_{4}^{\mathrm{int}}=-\left(\frac{1}{64}+\frac{1}{12 \sqrt{3}}\right) \frac{a_{s}}{\lambda}+O\left(\frac{a_{s}}{\lambda}\right)^{2} .
\end{gathered}
$$

To derive these expressions, we have adopted the results of SU(2) Fermi gas, i.e., $Q_{1,2}, Q_{1,3}$, etc., [60]. As expected, the dimensionless $b_{n}$ must be a power series of the dimensionless parameter $a_{s} / \lambda$. Using $\Omega$, we obtain both the total particle number and the contact:

$$
\begin{gathered}
N N_{0}=-\frac{\partial \Omega}{\partial \mu}=-\frac{\partial \Omega}{\partial z} \frac{\partial z}{\partial \mu}=-\frac{\partial \Omega}{\partial z} \frac{z}{k_{B} T}, \\
\frac{N \hbar^{2}}{8 \pi m} \mathcal{C}_{0}=-\frac{\partial \Omega}{\partial a_{s}^{-1}},
\end{gathered}
$$

both of which can be expressed in power series of $z$. Eliminating $z$, we obtain the contact as a function of $N, N_{0}$, and $T$ :

$$
\mathcal{C}_{0}^{(4)}=\mathcal{C}_{0}^{(2)} f_{F}\left(\frac{T}{T_{F}} ; k_{F} a_{s}\right)
$$

$$
\begin{aligned}
& f_{F}\left(\frac{T}{T_{F}} ; k_{F} a_{s}\right) \\
& =\left[1-\left(\frac{1}{9 \sqrt{3}}-\frac{1}{48}\right)\left(T / T_{F}\right)^{-3}\right. \\
& +\left(\frac{1}{108 \sqrt{6}}+\frac{1}{576 \sqrt{2}}-\frac{5}{31104}-\frac{1}{216 \sqrt{3}}\right)\left(T / T_{F}\right)^{-6} \\
& +\frac{(N-1) k_{F} a_{s}}{12 \sqrt{2 \pi}}\left(T / T_{F}\right)^{-5 / 2}+2\left(\frac{(N-1) k_{F} a_{s}}{12 \sqrt{2 \pi}}\right)^{2}\left(T / T_{F}\right)^{-5} \\
& \left.-(N-1) \frac{k_{F} a_{s}}{2 \sqrt{\pi}}\left(\frac{7}{108 \sqrt{6}}-\frac{1}{72 \sqrt{2}}\right)\left(T / T_{F}\right)^{-11 / 2}+\cdots\right]
\end{aligned}
$$

which is accurate up to $\left(T / T_{F}\right)^{-6}$. This function requires us to include contributions up to $b_{4}$ in Eq. (A32) and up to $b_{3}$ in Eq. (A31). Here, the Fermi temperature is a function of $N_{0}, T_{F}=\hbar \bar{\omega}\left(6 N_{0}\right)^{1 / 3} / k_{B}$, and $a_{s} / \lambda$ is replaced by $\left(k_{F} a_{s} / 2 \sqrt{\pi}\right) \sqrt{T / T_{F}} \cdot \mathcal{C}_{0}^{(2)}$ is the previously obtained contact in the second-order virial expansion, as shown in Eq. (A12). Since $\mathcal{C}_{0}^{(2)} \sim(N-1)$, the last three terms in the above equation lead to higher powers of $N$ in the scalings of the contact with $N$. However, all of them are much smaller than the other terms due to the smallness of $k_{F} a_{s}$ and $a_{s} / \lambda$. Using our experimental parameters, the total correction from these $(N-1)$-dependent terms for $N=6$ is up to $13 \%, 10 \%$, and $5 \%$ for $T / T_{F}=0.55,0.69$, and 1 , respectively. Thus, $\mathcal{C}=\left[\mathcal{C}_{0} /(2 \pi)^{3} N_{0} k_{F}\right]$ scales with $(N-1)$ in our experiments.

The slope of the linear scaling of $\mathcal{C}$ with $N-1$ is temperature dependent. Theoretical results plotted in Fig. 9 show that the slope increases with decreasing $T$. This result is also observed in experiments. As shown in Fig. 3(b), our experimental resolution is not able to distinguish the small curvatures, which come from the small high-order corrections to the linear scaling of $\mathcal{C}$ with $N-1$. As such, we use a linear fitting to obtain the slope. Again, the theoretical results of the slope are about 6-9 times of the 


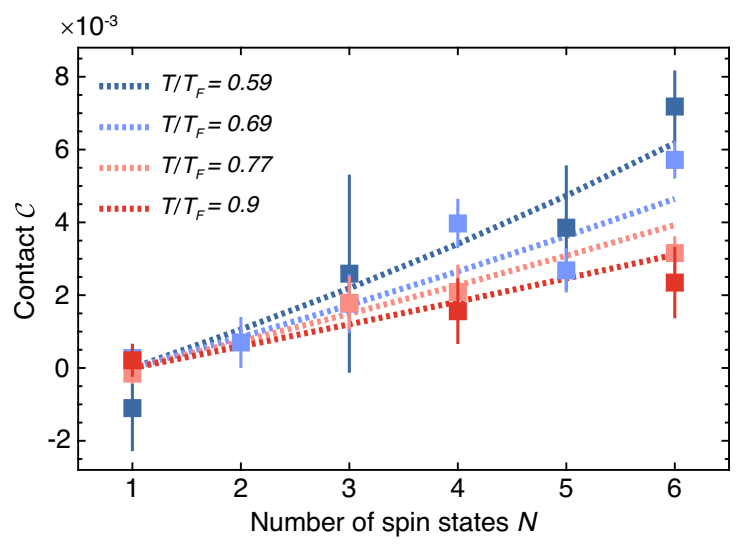

FIG. 9. Contact as a function of spin component $N$ at different temperatures $T / T_{F}$. Experimental results are denoted by boxes. Dotted lines represent theoretical results including corrections up to fourth-order virial expansion. All theoretical curves include a factor of 7.5 .

experimentally observed ones due to the expansion dynamics, as explained in the main text.

If we keep only contributions up to $b_{3}$ in Eq. (A32) and $b_{2}$ in Eq. (A31),

$$
\begin{aligned}
\mathcal{C}_{0}^{(3)}= & \mathcal{C}_{0}^{(2)}\left[1-\left(\frac{1}{9 \sqrt{3}}-\frac{1}{48}\right)\left(T / T_{F}\right)^{-3}\right. \\
& \left.+\frac{(N-1) k_{F} a_{s}}{12 \sqrt{2 \pi}}\left(T / T_{F}\right)^{-5 / 2}+\cdots\right]
\end{aligned}
$$

Both results are presented in Fig. 3 of the main text.

\section{High-order virial expansions for bosons}

The above analysis can be applied to bosons as well:

$$
\begin{aligned}
& \mathcal{C}_{B}^{(4)}=\mathcal{C}_{B}^{(2)} f_{B}\left(\frac{T}{T_{F}} ; k_{F} a_{s}\right), \\
& f_{B}\left(\frac{T}{T_{F}} ; k_{F} a_{s}\right) \\
& =\left[1+\left(\frac{1}{9 \sqrt{3}}-\frac{1}{48}\right) N\left(T / T_{F}\right)^{-3}\right. \\
& +\left(\frac{1}{108 \sqrt{6}}+\frac{1}{576 \sqrt{2}}-\frac{5}{31104}-\frac{1}{216 \sqrt{3}}\right) N^{2}\left(T / T_{F}\right)^{-6} \\
& +\frac{k_{F} a_{s}}{6 \sqrt{2 \pi}} N^{5 / 6}\left(T / T_{F}\right)^{-5 / 2}+\frac{\left(k_{F} a_{s}\right)^{2}}{36 \pi} N^{5 / 3}\left(T / T_{F}\right)^{-5} \\
& \left.+\left(\frac{7}{54 \sqrt{6}}-\frac{1}{36 \sqrt{2}}\right) \frac{k_{F} a_{s}}{2 \sqrt{\pi}} N^{11 / 6}\left(T / T_{F}\right)^{-11 / 2} \ldots\right]
\end{aligned}
$$

Here, $\mathcal{C}_{B}^{(2)}$ is the previously obtained contact of bosons in the second-order virial expansion, as shown in Eq. (A17).
$T_{F}$ is defined to be the Fermi temperature of the corresponding Fermi gas of a single component, $T_{F}=$ $\hbar \bar{\omega}\left(6 N_{0}\right)^{1 / 3} / k_{B}$, for convenience of comparison. Using the results of both bosons and $\mathrm{SU}(N)$ fermions, we obtain

$$
\mathcal{C}_{\mathrm{SU}(N)}^{(4)}=N \mathcal{C}_{0}^{(4)}=\alpha\left(\frac{T}{T_{F}} ; k_{F} a_{s}\right)\left(1-\frac{1}{N}\right) \mathcal{C}_{B}^{(4)},
$$

where $\quad \alpha\left[\left(T / T_{F}\right) ; k_{F} a_{s}\right]=\left[f_{B}\left(T / T_{F} ; k_{F} a_{s}\right) / f_{F}\left(T / T_{F}\right.\right.$, $\left.k_{F} a_{s}\right)$ ]. The virial expansion works for $\mathrm{SU}(N)$ fermions and bosons in the temperature regimes $T \gtrsim 0.55 T_{F}$ and $T \gtrsim 0.55 N^{1 / 3} T_{F}$, respectively. At $T \gg 0.55 N^{1 / 3} T_{F}$, both $f_{F}\left[\left(T / T_{F}\right) ; k_{F} a\right]$ and $f_{B}\left[\left(T / T_{F}\right) ; k_{F} a\right]$ can be well approximated by 1 . As such, $\mathcal{C}_{\mathrm{SU}(N)}^{(4)} \rightarrow \mathcal{C}_{B}^{(4)}$ when $N \rightarrow \infty$.

[1] S. Weinberg, The Quantum Theory of Fields (University of Texas, Austin, 2000).

[2] T. Giamarchi, Quantum Physics in One Dimension (Clarendon, Oxford, 2003).

[3] Z.X. Li, A. Vaezi, C. B. Mendl, and H. Yao, Numerical Observation of Emergent Spacetime Supersymmetry at Quantum Criticality, Sci. Adv. 4, eaau1463 (2018).

[4] A. Cherman and D. Dorigoni, Large $N$ and Bosonization in Three Dimensions, J. High Energy Phys. 10 (2012) 173.

[5] B. Paredes, A. Widera, V. Murg, O. Mandel, S. Fölling, I. Cirac, G. V. Shlyapnikov, T. W. Hänsch, and I. Bloch, Tonks-Girardeau Gas of Ultracold Atoms in an Optical Lattice, Nature (London) 429, 277 (2004).

[6] T. Kinoshita, T. Wenger, and D. S. Weiss, Observation of a One-Dimensional Tonks-Girardeau Gas, Science 305, 1125 (2004).

[7] C. N. Yang and Y.-Z. You, One-Dimensional w-Component Fermions and Bosons with Repulsive Delta Function Interaction, Chin. Phys. Lett. 28, 020503 (2011).

[8] X.-W. Guan, Z.-Q. Ma, and B. Wilson, One-Dimensional Multicomponent Fermions with $\delta$-Function Interaction in Strong- and Weak-Coupling Limits: $\kappa$-Component Fermi Gas, Phys. Rev. A 85, 033633 (2012).

[9] X.-J. Liu and H. Hu, Collective Mode Evidence of HighSpin Bosonization in a Trapped One-Dimensional Atomic Fermi Gas with Tunable Spin, Ann. Phys. (Amsterdam) 350, 84 (2014).

[10] Y. Jiang, P. He, and X.-W. Guan, Universal Low-Energy Physics in 1D Strongly Repulsive Multi-component Fermi Gases, J. Phys. A 49, 17 (2016).

[11] H. H. Jen and S.-K. Yip, Spin-Incoherent Luttinger Liquid of One-Dimensional $S U(\kappa)$ Fermions, Phys. Rev. A 98, 013623 (2018).

[12] X.-W. Guan, M. T. Batchelor, and C. Lee, Fermi Gases in One Dimension: From Bethe Ansatz to Experiments, Rev. Mod. Phys. 85, 1633 (2013).

[13] J. Decamp, J. Jünemann, M. Albert, M. Rizzi, A. Minguzzi, and P. Vignolo, High-Momentum Tails as MagneticStructure Probes for Strongly Correlated SU $(\kappa)$ Fermionic Mixtures in One-Dimensional Traps, Phys. Rev. A 94, 053614 (2016). 
[14] E. K. Laird, Z.-Y. Shi, M. M. Parish, and J. Levinsen, $\mathrm{SU}(N)$ Fermions in a One-Dimensional Harmonic Trap, Phys. Rev. A 96, 032701 (2017).

[15] G. Pagano, M. Mancini, G. Cappellini, P. Lombardi, F. Schäfer, H. Hu, X.-J. Liu, J. Catani, C. Sias, M. Inguscio et al., A One-Dimensional Liquid of Fermions with Tunable Spin, Nat. Phys. 10, 198 (2014).

[16] S. Tan, Energetics of a Strongly Correlated Fermi Gas, Ann. Phys. (Amsterdam) 323, 2952 (2008).

[17] S. Tan, Large Momentum Part of a Strongly Correlated Fermi Gas, Ann. Phys. (Amsterdam) 323, 2971 (2008).

[18] S. Tan, Generalized Virial Theorem and Pressure Relation for a Strongly Correlated Fermi Gas, Ann. Phys. (Amsterdam) 323, 2987 (2008).

[19] G. B. Partridge, K. E. Strecker, R. I. Kamar, M. W. Jack, and R. G. Hulet, Molecular Probe of Pairing in the BEC-BCS Crossover, Phys. Rev. Lett. 95, 020404 (2005).

[20] F. Werner, L. Tarruell, and Y. Castin, Number of ClosedChannel Molecules in the BEC-BCS Crossover, Eur. Phys. J. B 68, 401 (2009).

[21] J. T. Stewart, J. P. Gaebler, T. E. Drake, and D. S. Jin, Verification of Universal Relations in a Strongly Interacting Fermi Gas, Phys. Rev. Lett. 104, 235301 (2010).

[22] E. D. Kuhnle, S. Hoinka, P. Dyke, H. Hu, P. Hannaford, and C. J. Vale, Crossover from $2 D$ to $3 D$ in a Weakly Interacting Fermi Gas, Phys. Rev. Lett. 106, 170402 (2011).

[23] R. J. Fletcher, R. Lopes, J. Man, N. Navon, R. P. Smith, M. W. Zwierlein, and Z. Hadzibabic, Two- and ThreeBody Contacts in the Unitary Bose Gas, Science 355, 377 (2017).

[24] S. Laurent, M. Pierce, M. Delehaye, T. Yefsah, F. Chevy, and C. Salomon, Connecting Few-Body Inelastic Decay to Quantum Correlations in a Many-Body System: A Weakly Coupled Impurity in a Resonant Fermi Gas, Phys. Rev. Lett. 118, 103403 (2017).

[25] C. Wu, J.-p. Hu, and S.-C. Zhang, Exact SO(5) Symmetry in the Spin-3/2 Fermionic System, Phys. Rev. Lett. 91, 186402 (2003).

[26] M. Hermele, V. Gurarie, and A. M. Rey, Mott Insulators of Ultracold Fermionic Alkaline Earth Atoms: Underconstrained Magnetism and Chiral Spin Liquid, Phys. Rev. Lett. 103, 135301 (2009).

[27] A. V. Gorshkov, M. Hermele, V. Gurarie, C. Xu, P. S. Julienne, J. Ye, P. Zoller, E. Demler, M. D. Lukin, and A. M. Rey, Two-Orbital SU(N) Magnetism with Ultracold Alkaline-Earth Atoms, Nat. Phys. 6, 289 (2010).

[28] M. A. Cazalilla and A. M. Rey, Ultracold Fermi Gases with Emergent SU(N) Symmetry, Rep. Prog. Phys. 77, 124401 (2014).

[29] S. Taie, R. Yamazaki, S. Sugawa, and Y. Takahashi, An SU (6) Mott Insulator of an Atomic Fermi Gas Realized by Large-Spin Pomeranchuk Cooling, Nat. Phys. 8, 825 (2012).

[30] C. Hofrichter, L. Riegger, F. Scazza, M. Höfer, D. R. Fernandes, I. Bloch, and S. Fölling, Direct Probing of the Mott Crossover in the SU(N) Fermi-Hubbard Model, Phys. Rev. X 6, 021030 (2016).

[31] H. Ozawa, S. Taie, Y. Takasu, and Y. Takahashi, Antiferromagnetic Spin Correlation of $S U$ (N) Fermi Gas in an Optical Superlattice, Phys. Rev. Lett. 121, 225303 (2018).
[32] X. Zhang, M. Bishof, S. L. Bromley, C. V. Kraus, M. S. Safronova, P. Zoller, A. M. Rey, and J. Ye, Spectroscopic Observation of SU(N)-Symmetric Interactions in Sr Orbital Magnetism, Science 345, 1467 (2014).

[33] G. Cappellini, M. Mancini, G. Pagano, P. Lombardi, L. Livi, M. S. de Cumis, P. Cancio, M. Pizzocaro, D. Calonico, F. Levi, C. Sias, J. Catani, M. Inguscio, and L. Fallani, Direct Observation of Coherent Interorbital Spin-Exchange Dynamics, Phys. Rev. Lett. 113, 120402 (2014).

[34] F. Scazza, C. Hofrichter, M. Hofer, P. C. De Groot, I. Bloch, and S. Folling, Observation of an Orbital Interaction-Induced Feshbach Resonance in Yb 173, Nat. Phys. 11, 514 (2015).

[35] C. He, Z. Ren, B. Song, E. Zhao, J. Lee, Y.-C. Zhang, S. Zhang, and G.-B. Jo, Collective Excitations in TwoDimensional SU $(N)$ Fermi Gases with Tunable Spin, Phys. Rev. Research 2, 012028(R) (2020).

[36] L. Sonderhouse, C. Sanner, R. B. Hutson, A. Goban, T. Bilitewski, L. Yan, W. R. Milner, A. Maria Rey, and J. Ye, Thermodynamics of a Deeply Degenerate $S U(N)$-Symmetric Fermi Gas, Nat. Phys. https://doi.org/10.1038/s41567-0200986-6 (2020).

[37] T. Fukuhara, Y. Takasu, M. Kumakura, and Y. Takahashi, Degenerate Fermi Gases of Ytterbium, Phys. Rev. Lett. 98, 030401 (2007).

[38] B. Song, C. He, S. Zhang, E. Hajiyev, W. Huang, X.-J. Liu, and G.-B. Jo, Spin-Orbit-Coupled Two-Electron Fermi Gases of Ytterbium Atoms, Phys. Rev. A 94, 061604(R) (2016).

[39] The near-field effect arising from the initial size of the atomic cloud does not significantly affect the measurement of high-momentum tails. An atomic cloud with a radius $R$ after time-of-flight expansion has a near-field effect $d k \simeq \mathrm{Rm} /\left(\hbar t_{\text {tof }}\right)$, where $t_{\text {tof }}$ is the expansion time. In our experiment, the near-field correction $d k / k \leq 0.03$ is negligible in the large- $k$ regime $\left(k>3 k_{F}\right)$ where the contact is measured.

[40] Bo Song, Chengdong He, Zejian Ren, Entong Zhao, Jeongwon Lee, and Gyu-Boong Jo, Effective Statistical Fringe Removal Algorithm for High-Sensitivity Imaging of Ultracold Atoms, Phys. Rev. Applied 14, 034006 (2020).

[41] H. Hu, X.-J. Liu, and P. D. Drummond, Universal Contact of Strongly Interacting Fermions at Finite Temperatures, New J. Phys. 13, 035007 (2011).

[42] M. Sun and X. Leyronas, High-Temperature Expansion for Interacting Fermions, Phys. Rev. A 92, 053611 (2015).

[43] T.-L. Ho and E. J. Mueller, High Temperature Expansion Applied to Fermions near Feshbach Resonance, Phys. Rev. Lett. 92, 160404 (2004).

[44] X.-J. Liu, H. Hu, and P. D. Drummond, Virial Expansion for a Strongly Correlated Fermi Gas, Phys. Rev. Lett. 102, 160401 (2009).

[45] D. A. Butts and D. S. Rokhsar, Trapped Fermi Gases, Phys. Rev. A 55, 4346 (1997).

[46] R. Chang, Q. Bouton, H. Cayla, C. Qu, A. Aspect, C. I. Westbrook, and D. Clément, Momentum-Resolved Observation of Thermal and Quantum Depletion in a Bose Gas, Phys. Rev. Lett. 117, 235303 (2016).

[47] C. Carcy, S. Hoinka, M. G. Lingham, P. Dyke, C. C. N. Kuhn, H. Hu, and C. J. Vale, Contact and Sum Rules in a Near-Uniform Fermi Gas at Unitarity, Phys. Rev. Lett. 122, 203401 (2019). 
[48] S. L. Zhang, M. Y. He, and Qi Zhou, Contact Matrix in Dilute Quantum Systems, Phys. Rev. A 95, 062702 (2017).

[49] S. M. Yoshida and M. Ueda, $p$-Wave Contact Tensor: Universal Properties of Axisymmetry-Broken p-Wave Fermi Gases, Phys. Rev. A 94, 033611 (2016).

[50] T. L. Ho and Q. Zhou, Squeezing out the Entropy of Fermions in Optical Lattices, Proc. Natl. Acad. Sci. U.S.A. 106, 6916 (2009).

[51] T. L. Ho and Q. Zhou, Universal Cooling Scheme for Quantum Simulation, arXiv:0911.5506.

[52] A. Mazurenko, C. S. Chiu, G. Ji, M. F. Parsons, M. KanászNagy, R. Schmidt, F. Grusdt, E. Demler, D. Greif, and M. Greiner, A Cold-Atom Fermi-Hubbard Antiferromagnet, Nature (London) 545, 462 (2017).

[53] B. Yang, H. Sun, C.-J. Huang, H.-Y. Wang, Y. Deng, H.-N. Dai, Z.-S. Yuan, and J.-W. Pan, Cooling and Entangling Ultracold Atoms in Optical Lattices, Science 369, 550 (2020).

[54] S.-G. Peng, C.-X. Zhang, S. Tan, and K. Jiang, Contact Theory for Spin-Orbit-Coupled Fermi Gases, Phys. Rev. Lett. 120, 060408 (2018).
[55] P. Zhang and N. Sun, Universal Relations for Spin-OrbitCoupled Fermi Gas near an s-Wave Resonance, Phys. Rev. A 97, 040701(R) (2018).

[56] J. Jie, R. Qi, and Peng Zhang, Universal Relations of an Ultracold Fermi Gas with Arbitrary Spin-Orbit Coupling, Phys. Rev. A 97, 053602 (2018).

[57] X.-J. Liu, Virial Expansion for a Strongly Correlated Fermi System and Its Application to Ultracold Atomic Fermi Gases, Phys. Rep. 524, 37 (2013).

[58] S. E. Gharashi, K. M. Daily, and D. Blume, Three s-WaveInteracting Fermions under Anisotropic Harmonic Confinement: Dimensional Crossover of Energetics and Virial Coefficients, Phys. Rev. A 86, 042702 (2012).

[59] T. Busch, B. G. Englert, K. Rzazewski, and M. Wilkens, Two Cold Atoms in a Harmonic Trap, Found. Phys. 28, 549 (1998).

[60] E. Marcelino, A. Nicolai, I. Roditi, and A. LeClair, Virial Coefficients for Trapped Bose and Fermi Gases beyond the Unitary Limit: An S-Matrix Approach, Phys. Rev. A 90, 053619 (2014). 